\title{
The Sulfinate-Sulfone Pummerer Rearrangement
}

Pummerer-Rearrangements are well-known to organic Sulfur chemists to yield internal redox products from $\mathrm{C}, \mathrm{H}$-acidic sulfoxides including desoxygenation of sulfur and oxygenation of $\alpha$-carbon-hydrogen bonds.

(DIA1)

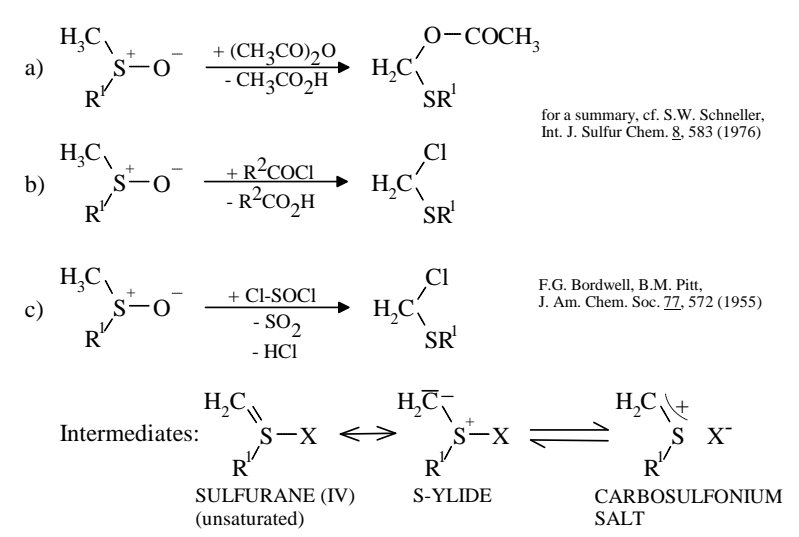

According to the reaction conditions the hydroxy group of hemithioacetals is replaced by chloride or acyloxy groups. Presumably sulfuranes (IV), sulfur ylides and carbosulfonium salts are reactive intermediates.

Since thionyl chloride may be regarded as chlorosulfinic acid chloride, it was of interest whether application of sulfinyl chlorides would lead to an introduction of ambident sulfinate nucleophiles.

(DIA2)

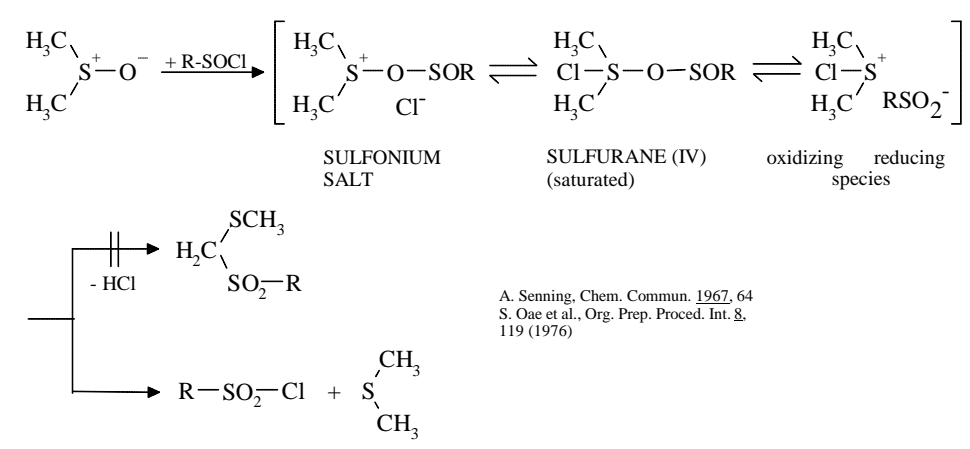

However, as has been mentioned in literature, this was not the case with DMSO, intermolecular oxygen transfer represented the main reaction pathway. This was particularly deplorable because sulfonyl thioethers as valuable agents for nucleophilic acylations were not available by this simple way. 
(DIA3)

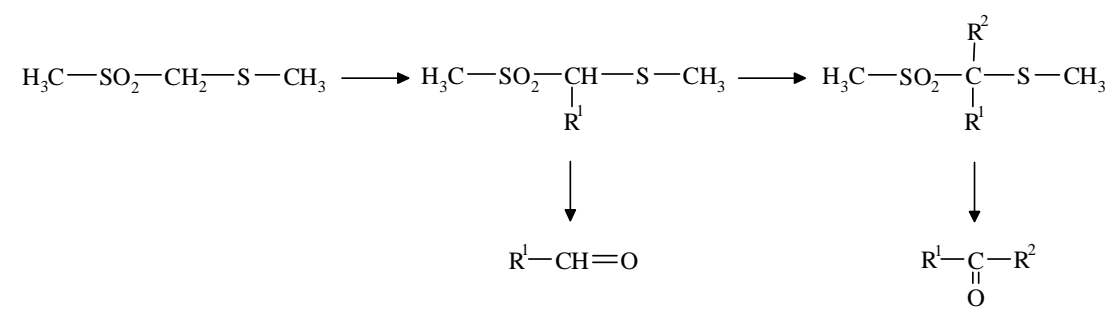

1) K. Schank, Methoden der organischen Chemie (Houben-Weyl), 4. Aufl., Bd. E11 (D. Klamann), S. 1286, Thieme, Stuttgart

2) K. Ogura, Pure \& Appl. Chem. 59,1033 (1987)
3) B. Wladislaw, L. Marzorati, Rev. Heteroatom Chem. (S. Oae) Vol. 9, MYU, Tokyo 1993

(DIA4)

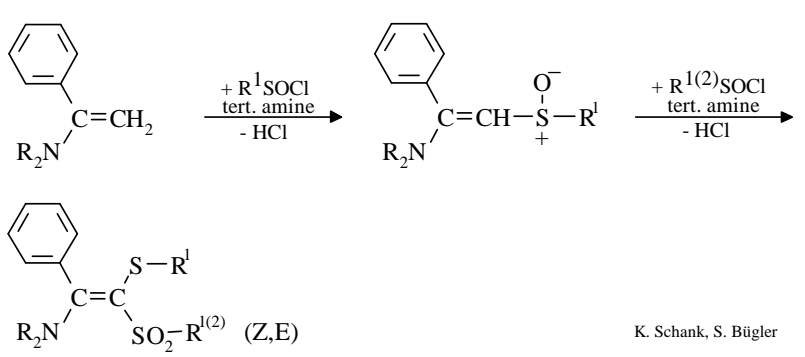

In order to modify the basic sulfoxide we converted $\alpha$-amino styrenes with sulfinylchlorides to get vinylogous sulfinamides. These conversions succeeded only in part because a secondary reaction, a desired sulfinate-sulfone Pummererreaction, ensued as fast as the first step.

(DIA5)

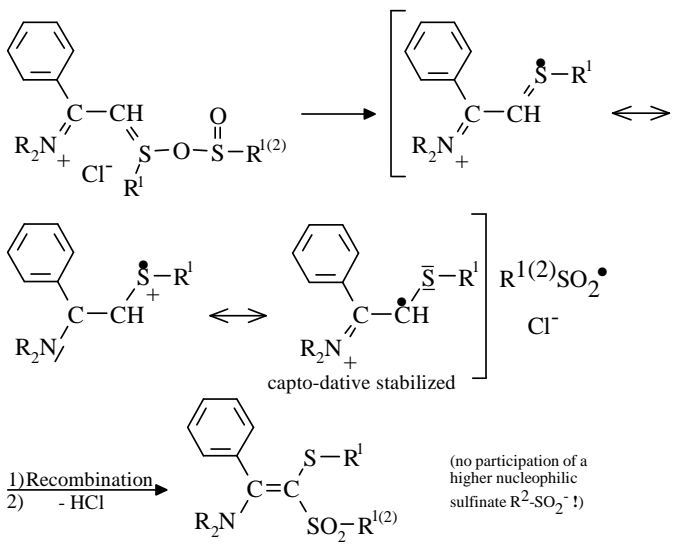

We propose the mechanism of this rearrangement to be rather radical than ionic like that of Pummerer reactions mentioned before. Our arguments are as follows: 1) After addition of 2 equivalents of sulfinyl chloride to the vinyl amine at $-78^{\circ} \mathrm{C}$ in presence of a tert. amine, a deep yellow color appeared which remained consistent during warming-up until $-40^{\circ} \mathrm{C}$. At that point, the yellow color disappeared 
suddenly and at the same time colorless amine hydrochloride precipitated from the solution. 2) Addition of sulfinate of higher nucleophilicity to the yellow solution did not lead to cross reaction products.

(DIA6)

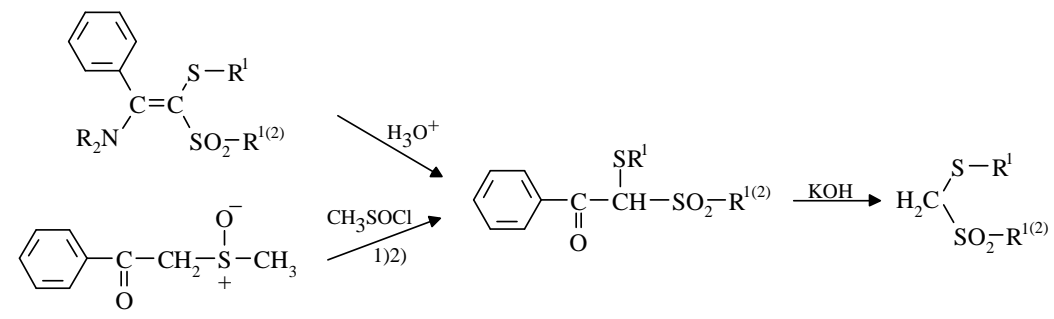

B-Oxosulfoxides via acylation of methylene active sulfoxides: 1) R. Kawecki, L. Kozerski, Tetrahedron 42, 1469 (1986)

Hydrolysis of the enamino function led to $\beta$-oxo sulfoxides which are known from literature via other reaction pathways. Acyl cleavage of B-oxo-sulfoxides led to the desired a-sulfonyl thioethers.

(DIA7)

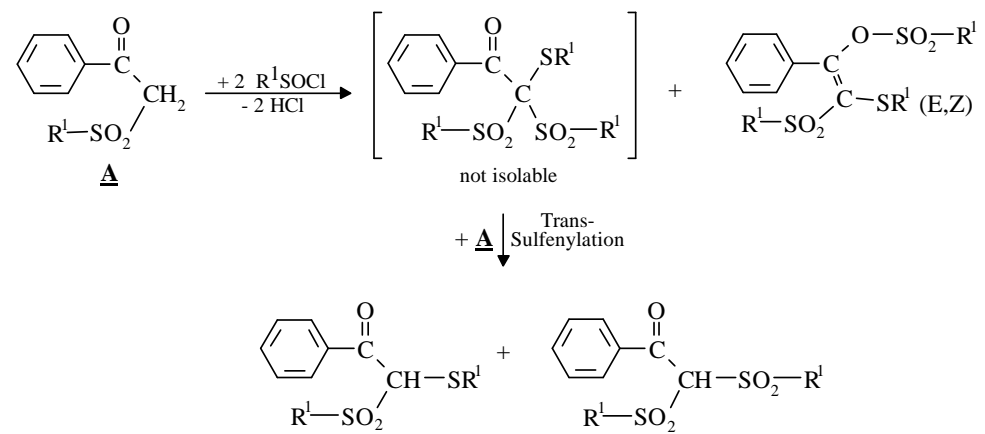

ß-Oxosulfones instead of $\beta$-oxosulfoxides reacted with sulfinylchlorides to show geminal di- thiofunctionalization. The resulting products, however, could not be isolated, they suffered as well isomerization to yield enolsulfonates as well transsulfenylations to yield acylated sulfonyl thioethers and acylated ß-disulfones. 
(DIA8)

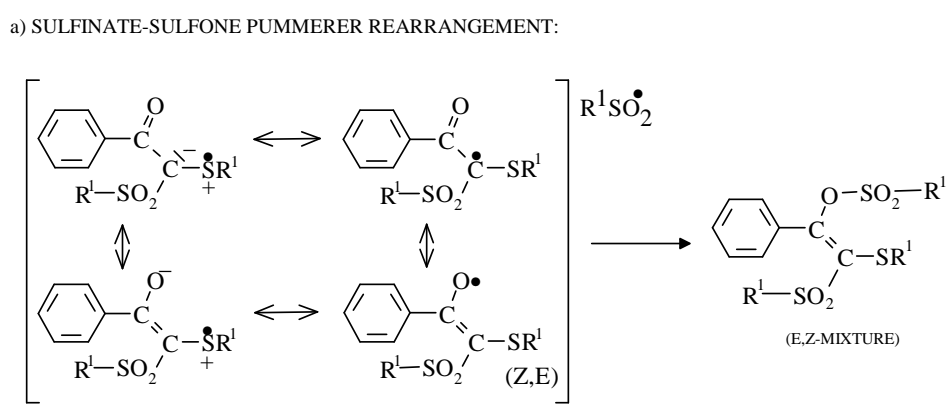

b) ENOLATE SULFONIC ESTERIFICATION:

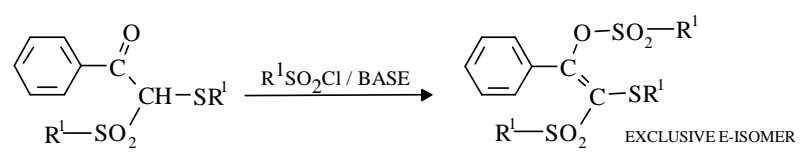

The obtained enolsulfonates showed to be Z,E-mixtures pointing to a similar radical mechanism as discussed before. The ionic conversion of acylated $\alpha$-sulfonyl thioether with sulfonyl chloride in presence of base afforded exclusively the E-isomers.

(DIA9)

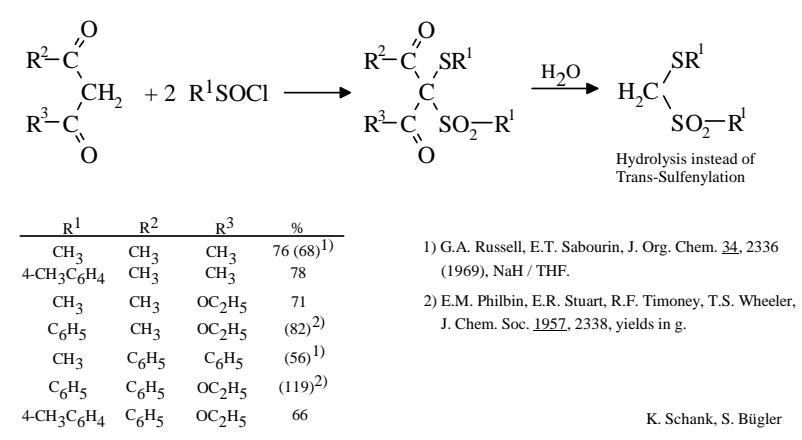

Since $\beta$-oxo-sulfones are representatives of methylene active compounds consequently B-dicarbonyl compounds were investigated under the same reaction conditions. Some of these conversions have been described in literature with open-chain $\beta$-dicarbonyl compounds, $\alpha$-sulfonyl thioether could be obtained via this sequence by a one-pot procedure in reasonable yields. 
(DIA10)

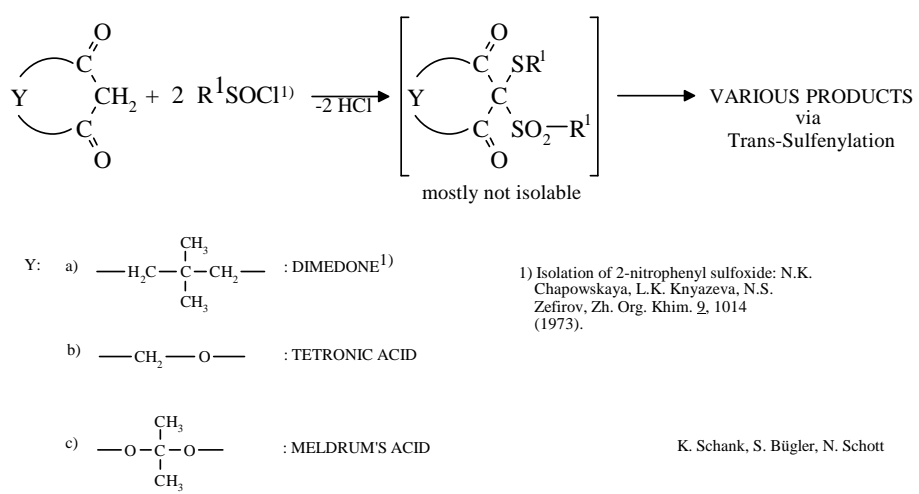

Using cyclic ß-dicarbonyl compounds, the intermediate dithiofunctionalization products showed a very high sulfenylating property as already mentioned in connection with ß-oxosulfones. No enol sulfonates were observed in these cases. (DIA11)

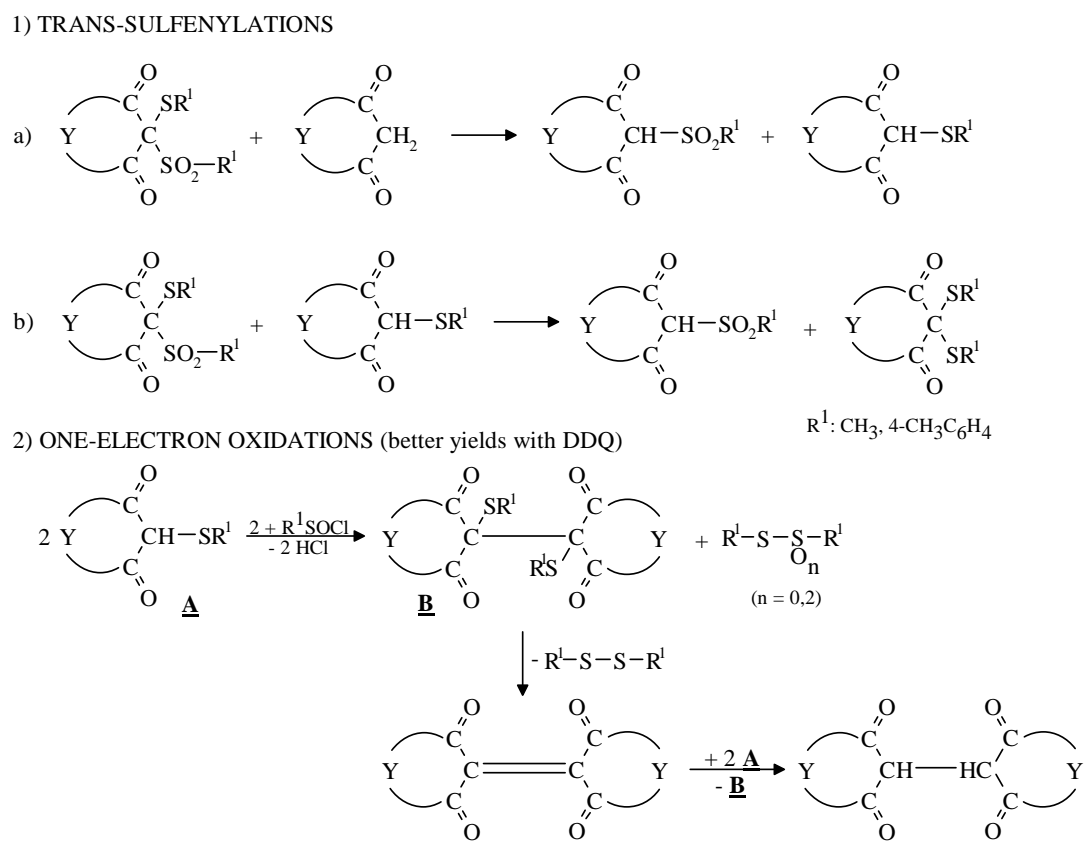

However, the trans-sulfenylations were accompanied in these cases by reaction products of secondary one-electron oxidations leading to preparatively interesting dimerizations and further redox-disproportionations. Thus, for example tetraacyl ethylenes and/or ethanes were easily obtainable by this way. 
(DIA12)

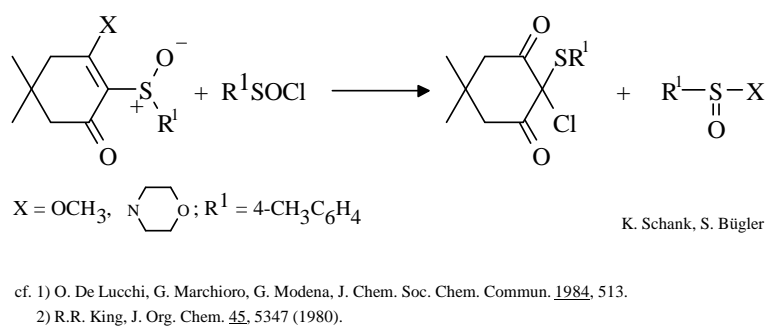

Further efforts in order to obtain the intermediates via a more smooth method starting from enol ethers or enamines from dimedone were fruitless. In these cases chloroorganothio dimedones as derivatives of the corresponding vicinal triketone were isolated together with the corresponding derivatives of sulfinic acid.

Excellent co-working of Dr. Bügler, Dr. Schott and Thomas Heisel should be emphasized at this place, I thank you for your kind attention. 